\title{
Crecimiento y mortalidad del pez Haemulon aurolineatum (Teleostei: Haemulidae) en el suroeste de la isla de Margarita, Venezuela
}

\author{
Edwis Bravo ${ }^{1}$, Nora Eslava² \& Leo González ${ }^{2}$ \\ 1. Escuela de Ciencias Aplicadas del Mar, Departamento de Acuacultura, Instituto de Investigaciones Científicas, Núcleo \\ de Nueva Esparta, Universidad de Oriente, Venezuela; edwisb@gmail.com \\ 2. Área de Biología y Recursos Pesqueros, Instituto de Investigaciones Científicas, Núcleo de Nueva Esparta, \\ Universidad de Oriente, Venezuela.
}

Recibido 28-V-2008. Corregido 10-VIII-200. Aceptado 11-IX-2008.

\begin{abstract}
Growth and mortality of the fish Haemulon aurolineatum (Teleostei: Haemulidae) from Southwest of Margarita Island, Venezuela. We analyzed the growth and natural mortality of the fish known locally as cují (H. aurolineatum) in the southwest of Margarita Island, July 2005 to June 2006. A sample of 1378 males and 1143 females from artisanal fishing vessels of Boca del Río was analyzed. The common relation for both sexes was expressed by the equation $\mathrm{P}=0.038 * \mathrm{~L}^{2.87}$. The asymptotic length $\left(\mathrm{L}_{\infty}\right)$ was estimated with Powell`s (1979) routine and Wetherall (1986), and the growth coefficient (k) with ELEFAN I - FISAT II (FAO-ICLARM). The modal progression analysis was used after decomposition of lengths frequency according to Bhattacharya, and the estimations of $\mathrm{L}_{\infty}$ and $\mathrm{k}$ optimized according to Gulland and Holt (1959). The growth curve was fitted to von Bertalanffy (1960); it is exponential, with accelerated growth in the first two years and less growth as the fish approaches maximal length. The rate of natural mortality was high $\left(\mathrm{M}=1.15\right.$ year $\left.^{-1}\right)$, and probably was caused by high depredation. Rev. Biol. Trop. 57 (1-2): 105-112. Epub 2009 June 30.
\end{abstract}

Key words: growth, mortality, Haemulon aurolineatum, Margarita Island, Venezuela.

El crecimiento de los peces puede considerarse como un incremento en longitud o peso, y es el resultado directo de procesos químicos, osmóticos y otros factores que contribuyen al aporte de material en el organismo, el cual es transferido a muchas partes del cuerpo (Tresierra y Culquichicón 1993). Los estudios de crecimiento poblacional de peces son esenciales para la mayor parte de los objetivos de la evaluación pesquera, tanto en las regiones tropicales como en las templadas, debido a que el crecimiento individual de los organismos constituye, a través del tiempo, la fuente de suministro de las capturas extraídas por una pesquería (Pauly 1983). La tasa de crecimiento biológico se relaciona con procesos tales como la maduración sexual, el riesgo de la depredación, la longevidad, y es fundamental para la cuantificación de la productividad del stock y exceso de producción (Sparre et al. 1997).

El método de frecuencia de longitudes se utiliza para realizar estimaciones del crecimiento, especialmente en peces tropicales de vida corta. Froese y Binohlan (2000) han demostrado que la distribución de frecuencia de tallas puede utilizarse para hacer una primera evaluación del estado de un stock. En los trópicos, los métodos basados en el análisis de datos sobre frecuencia por clases de longitud han encontrado aplicación más amplia que el estudio de las estructuras esqueléticas; en tanto que las investigaciones basadas en el método de marcaje han sido por lo general poco utilizadas.

La fluctuación en la abundancia de los recursos pesqueros como consecuencia de las 
condiciones biológicas, ambientales y de pesca afectan el reclutamiento, lo que amerita información de índole periódica de los parámetros de crecimiento, mortalidad y reclutamiento, indispensables en la evaluación, y toma de decisiones con enfoque precautorio, en la administración de su pesquería (González y Eslava 1999).

El cují ( $H$. aurolineatum) está entre los peces más capturados por la pesca deportiva, comercial y de subsistencia en el Atlántico Centro Occidental (Darcy 1983). Es una de las especies más abundantes en los alrededores de las islas de Margarita, Coche y Cubagua, Venezuela (Cervigón 1994); es capturado principalmente con redes, trampas y anzuelos (Carpenter 2002), y con red de arrastre en la pesca industrial del camarón como fauna acompañante (Giménez et al. 1993). Dado que en el Oriente de Venezuela, el cují ocupa un lugar importante en las capturas comerciales y tiene una gran demanda para el consumo en fresco (Kossowski 1985), así como por ser un recurso asociado pesquerías multiespecíficas, se considero oportuno determinar el crecimiento y la mortalidad del cují ( $H$. aurolineatum) del suroeste de la isla de Margarita, Venezuela, y de esta forma contribuir al conocimiento de la biología pesquera de esta especie, la cual su captura, junto con el resto del grupo de los corocoros (Haemulidos), ha venido incrementándose en los últimos años.

\section{MATERIALES Y MÉTODOS}

Se realizaron colectas mensuales de cují (H. aurolineatum), entre julio de 2005 y junio de 2006, provenientes de la flota artesanal de Boca del Río (1050’38" - 1050'45" N; $\left.64^{\circ} 07^{\prime} 29^{\prime \prime}-64^{\circ} 07^{\prime} 41^{\prime \prime} \mathrm{W}\right)$, en el suroeste de la Isla de Margarita (Fig. 1). Se registraron datos de longitud total (LT), según lo sugerido por Holden y Raitt (1975), mediante un ictiómetro marca Wildco $(0.01 \mathrm{~cm})$ y el peso total $(\mathrm{P}) \mathrm{de}$

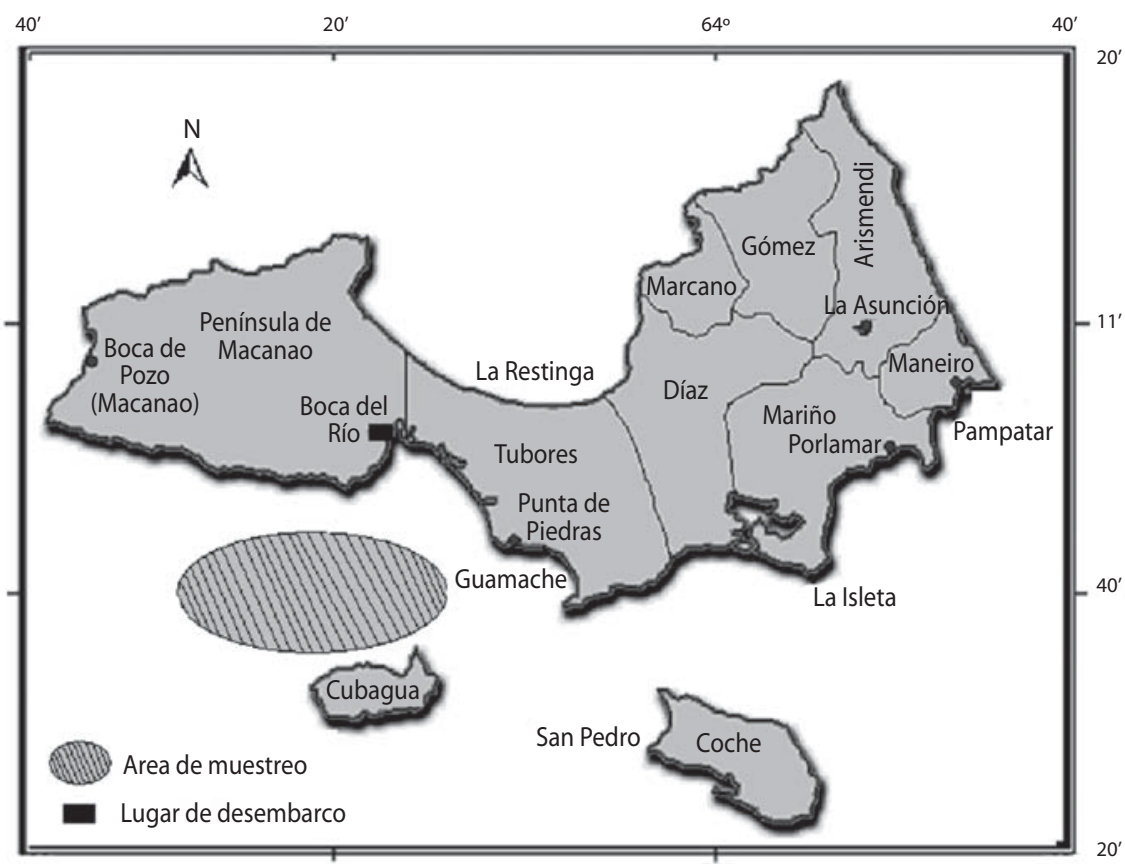

Fig. 1. Ubicación geográfica de la zona de muestreo.

Fig. 1. Geographic location of the sampling area. 
cada individuo mediante el uso de una balanza electrónica marca Sartorius (0.01 g).

La relación entre la longitud y el peso se estimó a través de la ecuación de alometría de Ricker (1975):

$\mathrm{P}=\mathrm{a}^{*} \mathrm{LT}^{\mathrm{b}}(1)$

donde $P$ es el peso total del pez sin eviscerar; $L T$ es la longitud total; $a$ es la intersección del eje de las ordenadas y $b$ es el exponente de la ecuación; ambas constantes de la referida ecuación fueron determinadas por mínimos cuadrados (Sokal y Rohlf 1975). Se determinó la existencia de diferencias significativas entre los coeficientes de machos y hembras ( $a$ y $b$ de la ecuación 1) y se probó la hipótesis del crecimiento isométrico (Ricker 1975) mediante una prueba t student (Zar 1996).

Los parámetros de crecimiento $\mathrm{L}_{\infty} \mathrm{y} \mathrm{k}$ de la ecuación de crecimiento de von Bertalanffy (1960) (ECVB) se estimaron mediante las distribuciones mensuales de frecuencia de tallas de machos y hembras con un ámbito de $1 \mathrm{~cm}$. En primer lugar, se estimó una longitud asintótica $\left(\mathrm{L}_{\infty}\right)$ preliminar aplicando el método de Powell (1979) y Wetherall (1986), que permitió determinar el coeficiente de crecimiento $(\mathrm{k})$ a través de la rutina ELEFAN I del Programa FAO-ICLARM Stock Assessment Tools (FISAT) (Gayanilo et al. 1996). Posteriormente se empleó el análisis de la progresión modal, previa descomposición de la frecuencia de longitudes según el método de Bhattacharya (1967), para optimizar las estimaciones de $\mathrm{L}_{\infty}$ y k según el procedimiento de Gulland y Holt (1959), incorporado en la misma rutina. Para obtener la curva de crecimiento en longitud del modelo de von Bertalanffy (1960), se calculó el $\mathrm{t}_{0}$ promedio de acuerdo a la fórmula anotada por Pauly (1984):

$$
\log \left(-\mathrm{t}_{0}\right)=-0.3922-0.2752 * \log \mathrm{L}_{\infty}(-1.038) * \log \mathrm{k}(2)
$$

Una vez calculados los parámetros de ECVB $\left(\mathrm{L}_{\infty}, \mathrm{k} \mathrm{y} \mathrm{t}_{0}\right)$, se establecieron las curvas de crecimiento ajustadas al modelo de von Bertalanffy (1960) en longitud y peso (Csirke
1980) hasta la edad limite, según las siguientes ecuaciones:

$$
\begin{aligned}
& \mathrm{L}_{\mathrm{t}}=\mathrm{L}_{\infty}\left\{1-\exp ^{[-\mathrm{k}(\mathrm{t}-\mathrm{t} 0)]}\right\} \\
& \mathrm{P}_{\mathrm{t}}=\mathrm{P}_{\infty}\left\{1-\exp ^{[-\mathrm{k}(\mathrm{t}-\mathrm{t} 0)]}\right\}^{\mathrm{b}}
\end{aligned}
$$

donde $\mathrm{L}_{\infty}=$ longitud asintótica; $\mathrm{k}=\mathrm{es}$ una constante que determina la tasa a la cual $\mathrm{L}_{t}$ se aproxima a $\mathrm{L}_{\infty} ; \mathrm{t}=$ edad del pez; $\mathrm{y} \mathrm{t}_{0}=\mathrm{la}$ edad hipotética a la cual la longitud media es cero si el pez hubiese crecido siempre de acuerdo a la ECVB. La edad límite se determinó según la siguiente ecuación (Taylor 1984):

$$
A_{0.95}=\frac{-\ln (1-0.95)}{k+t_{o}}
$$

donde: $\mathrm{A}_{0,95}$ es la edad límite o tiempo requerido para que el pez alcance el $95 \%$ de su longitud máxima teórica $\left(\mathrm{L}_{\infty}\right)$. Se validaron los parámetros de crecimiento comparándolo con los obtenidos por otros autores, mediante el Phi prima $\left(\phi^{\prime}\right)$ a través de la ecuación de Pauly y Munro (1984):

$$
\phi^{\prime}=\log \mathrm{k}+2 * \log \mathrm{L}_{\infty}(6)
$$

La mortalidad natural (M) se calculó a través de la ecuación empírica de Pauly (1983) para una temperatura superficial media del agua (T) de $27{ }^{\circ} \mathrm{C}$ (González 1985), en función de la siguiente ecuación:

$\log \mathrm{M}=-0.00660 .279 \log \mathrm{L}_{\infty}+0.6543 \log \mathrm{k}+0.4632 \log \mathrm{T}$

\section{RESULTADOS}

Se muestreo un total de 2521 ejemplares, desde julio de 2005 hasta junio de 2006. Los machos fueron más numerosos que las hembras (54.66\% y $45.34 \%$, respectivamente). La estructura de tallas de la muestra estuvo comprendida por ejemplares de $12.2 \mathrm{~cm}$ hasta $23.6 \mathrm{~cm}$ de LT. El peso total de los machos estuvo comprendido entre 25.83 y 179.92 g y las hembras entre 24.19 y $166.83 \mathrm{~g}$; ambos sexos estuvieron bien representados en todas las clases de tallas (Fig. 2). 


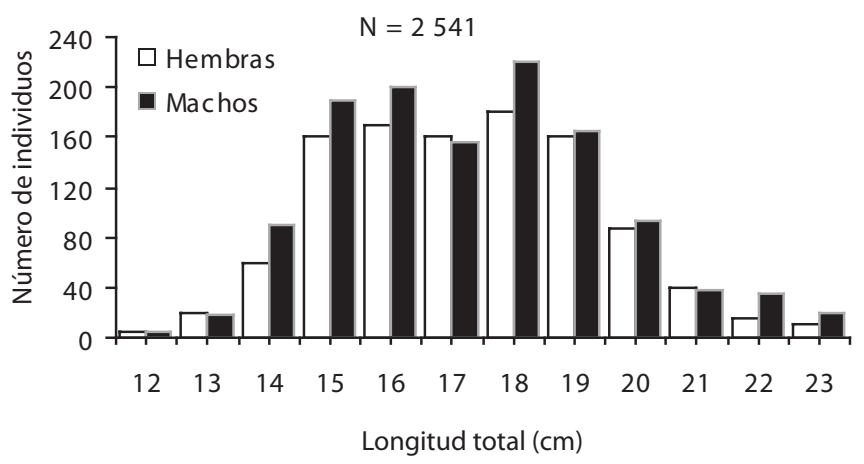

Fig. 2. Distribución de longitudes del cují (H. aurolineatum) del suroeste de la isla de Margarita, Venezuela.

Fig. 2. Length distribution of the cují (H. aurolineatum) from Southwest of Margarita island, Venezuela.

Los valores de la pendiente (b) de machos, hembras y sexos combinados fueron significativamente menores a $3(\mathrm{p}<0.05 ; \mathrm{Fs}=37.26)$. Las pendientes para machos y hembras no mostraron diferencias significativas $(\mathrm{p}>0.05$; ts $=-1.69)$, ni los interceptos ( $\mathrm{p}>0.05 ; \mathrm{ts}=-1.01)$, por lo que se estableció una relación común para ambos sexos, la cual quedó expresada mediante el modelo $\mathrm{P}=0.038^{*} \mathrm{LT}^{2.87}$ (Fig. 3). $H$. aurolineatum exhibió un crecimiento alométrico negativo para sexos combinados, lo cual significa que la especie tiende a ser más delgada a medida que aumenta en longitud.

Mediante la aplicación del método de Powell (1979) y Wetherall (1986), se hizo una primera estimación de la longitud asintótica $\mathrm{L}_{\infty}$ igual a $24.2 \mathrm{~cm}$, la cual fue utilizada como dato de entrada en el ELEFAN 1, obteniendo una primera estimación de $\mathrm{k}=0.48 \mathrm{año}^{-1}$. Se aplicó la rutina de Gulland y Holt (1959) proporcionando valores adicionales de $\mathrm{L}_{\infty} \mathrm{y}$ $\mathrm{k}$, que fueron descartados porque el valor del coeficiente de crecimiento estimado fue considerablemente alto $\left(\mathrm{k}=6.52 \mathrm{año}^{-1}\right)$ no estando acorde con la biología de la especie. El valor del $\mathrm{t}_{0}$ calculado fue de 0.29 años. La curva de crecimiento en longitud ajustada al modelo de von Bertalanffy (1960), describió una curva de tipo exponencial, donde se observó un crecimiento acelerado hasta los dos años de edad,

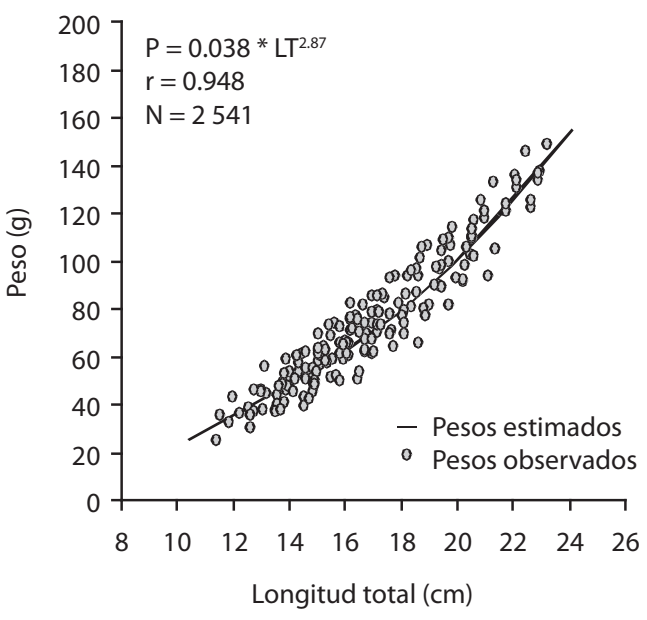

Fig. 3. Relación longitud-peso del cují (H. aurolineatum), del suroeste de la isla de Margarita.

Fig. 3. Length-weight relationship of cují (H. aurolineatum) from Southwest of Margarita island, Venezuela.

pero que luego se hace más lento a medida que alcanza la longitud máxima (Fig. 4). La curva de crecimiento en peso, presentó un patrón diferente, en las etapas tempranas de vida el incremento en peso fue lento, hasta llegar a los 2 y/o 3 años de edad, donde el crecimiento sufre un cambio, produciéndose una inflexión en la que la especie va aumentando su peso 


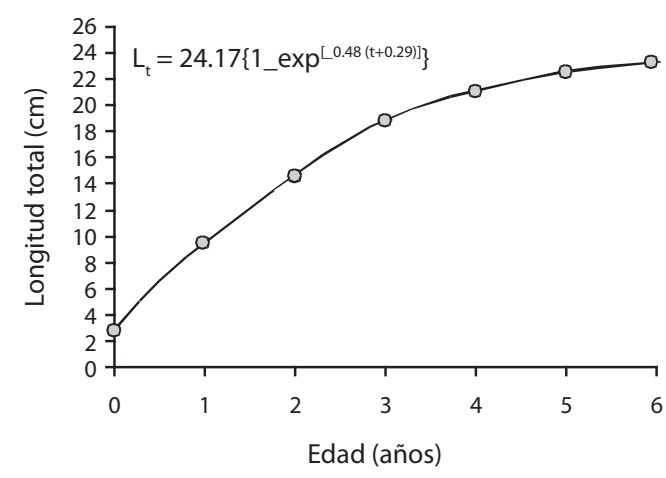

Fig. 4. Curva de crecimiento estimada en longitud del cují, Haemulon aurolineatum ajustada al modelo de von Bertalanffy (1960).

Fig. 4. Growth curve in length of cují (H. aurolineatum) fitted to model of von Bertalanffy (1960).

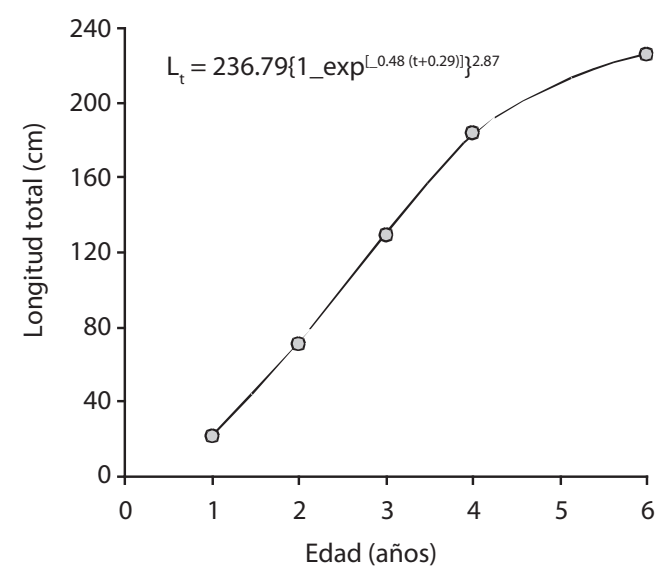

Fig. 5. Curva de crecimiento estimada en peso del cují, Haemulon aurolineatum ajustada al modelo de von Bertalanffy (1960).

Fig. 5. Growth curve in weight of cují (H. aurolineatum) fitted to model of von Bertalanffy (1960).

hasta alcanzar progresivamente su peso asintótico (Fig. 5).

Se compararon diferentes curvas de crecimiento de $H$. aurolineatum estimados en otros estudios y en diferentes localidades, utilizando el parámetro $\left(\phi^{\prime}\right)$ Phi prima (Pauly y Munro 1983, Munro y Pauly 1984). Los resultados de los valores del $\phi^{\prime}$ indican que son muy similares (Coeficiente de variación 5.4\%) (Cuadro 1). Con los parámetros de crecimiento obtenidos, se estimó el valor del coeficiente de la mortalidad natural, que fue $\mathrm{M}=1.15 \mathrm{año}^{-1}$.

\section{DISCUSIÓN}

El ámbito de longitudes encontradas en el presente trabajo, tanto para machos como para hembras coincide parcialmente con lo reportado para la misma especie por diferentes autores. De Lima et al. (2004) encontraron ejemplares con tallas entre 10.5 y $23.5 \mathrm{~cm}$ en las costas de Pernambuco, Brasil. Konchina (1976) señala que $H$. aurolineatum en las costas del Atlántico alcanza $26 \mathrm{~cm}$ de longitud total. En las costas Venezolanas, varios autores registraron diferentes tallas de $H$. aurolineatum, entre ellos, Kossowski (1985) registró ejemplares entre 13.2 y $23.2 \mathrm{~cm}$ y Cervigón (1994), quien encontró especimenes hasta 24.5 $\mathrm{cm}$, en los Frailes. Por otro lado, Manooch y Barans (1982), capturaron un ejemplar de 28.9 $\mathrm{cm}$ en la costa suroeste de los Estados Unidos de Norteamérica, siendo la máxima longitud registrada hasta la fecha.

La ausencia de tallas menores (LT $<12$ $\mathrm{cm})$ se debe, probablemente a la selectividad de los artes de pesca utilizados en la captura comercial, y a la disponibilidad del recurso en el área de pesca. Es probable que los jóvenes de $H$. aurolineatum se encuentren en áreas de crianza de aguas tranquilas y someras, o en zonas de manglares y/o lagunas costeras cercanas al área de pesca. Además se ha reportado que los jóvenes de $H$. aurolineatum se dirigen a los estuarios en búsqueda de abrigo y para la maduración de las gónadas, permaneciendo fuera del área de pesca, hasta alcanzar cierta talla en la cual se reclutan al stock de adultos (Lowe-McConnell 1999).

El valor de $b$ encontrado en el presente estudio resultó ser menor a 3 , evidenciando un crecimiento alométrico negativo o minorante, lo que significa que el pez es menos pesado para la longitud que tiene (Tresierra y Culquichicón 1993). Esto concuerda con lo señalado para la 
CUADRO 1

Parámetros de crecimiento de Haemulon aurolineatum estimados por diferentes autores

TABLE 1

Growth parameters estimated of Haemulon aurolineatum from different authors

$\begin{array}{lccccl}\text { Localidad } & \mathrm{L}_{\infty}(\mathrm{cm}) & \mathrm{k}\left(\mathrm{año}^{-1}\right) & \phi^{\prime} & \mathrm{T}\left({ }^{\circ} \mathrm{C}\right) & \text { Fuente } \\ \text { Pernambuco, Brasil } & 24.17 & 0.23 & 2.13 & 26.0 & \text { De Lima et al. }(2004) \\ \text { Arrecifes, Jamaica } & 23,0 & 0.35 & 2.27 & 27.0 & \text { Munro (1974) } \\ \text { Campeche, México } & 26.7 & 0.21 & 2.18 & 21.5 & \text { Sokolova (1965) } \\ \text { Carolina, USA } & 31.0 & 0.22 & 2.33 & 24.0 & \text { Manoch y Barans (1982) } \\ \text { Margarita, Venezuela } & 24.2 & 0.48 & 2.44 & 27.0 & \text { Este trabajo }\end{array}$

misma especie por Parra (1996) quien encontró un valor de $b$ de $2.89(\mathrm{~N}=433)$ en la isla de Cubagua (Venezuela), y difiere a los reportados por Manooch y Barans (1982) quienes indican un valor de b de $3.09(\mathrm{~N}=70)$ en el suroeste de las costas de Estados Unidos (USA), y Kossowski (1985) quien encontró un valor de b de 3.05 ( $\mathrm{N}=537)$ en Pampatar, isla de Margarita (Venezuela).

Los parámetros de crecimiento obtenidos por el método de frecuencia de tallas $\left(L_{\infty}=24.2\right.$ $\mathrm{cm}, k=0.48 \mathrm{año}^{-1}$ y $\left.t_{0}=-0.289\right)$ difieren ligeramente a los reportados por De Lima et al. (2004), estimados mediante el uso de estructuras duras. Estos autores señalan que H. aurolineatum en las costas de Pernambuco, Brasil, presenta un crecimiento lento $\left(L_{\infty}=24.17 \mathrm{~cm}\right.$ y $k=0.2336$ año $\left.^{-1}\right)$. Las variaciones de estos parámetros podrían ser debidas a diferencias de crecimiento por habitar en distintas latitudes que presentan características ambientales, como la temperatura y el fotoperiodo, propias de dichas regiones, las cuales pueden variar estacionalmente y están correlacionadas con los cambios en la abundancia y calidad del alimento (ciclos de producción del mar); los regímenes de explotación a los que están sometidos o por la metodología utilizada.

Los valores de Phi prima ( $\phi$ ') calculados para la especie de los parámetros de crecimiento estimados en trabajos previos y en este estudio, están distribuidos normalmente (Cuadro 1). Pauly y Munro (1984) encontraron que las especies en una misma familia tienen valores similares, de $\phi$ ' y calcularon este parámetro para gran número de especies encontrando que, dentro de una familia, los valores de $\phi$ ' están normalmente distribuidos. El valor del coeficiente de variación del índice de crecimiento $\phi$, indica similitud en los parámetros de crecimiento de $H$. aurolineatum obtenidos por diferentes autores en distintas localidades. Por su parte, Pauly (1979) y Sparre et al. (1994), establecen que el coeficiente de variación no debe exceder del $4 \%$ para poder asumir que los resultados son estadísticamente similares.

El valor de la tasa de mortalidad natural obtenido $\left(\mathrm{M}=1.15 \mathrm{año}^{-1}\right)$ induce a pensar que $H$. aurolineatum presenta una elevada mortalidad natural, probablemente, relacionada a la alta depredación, así como a las altas temperaturas del agua, típicas de las regiones tropicales, que aceleran los procesos biológicos, tal como lo establece Pauly (1979), cuando describe M como función de $\mathrm{k}, \mathrm{L}_{\infty} \mathrm{y}$ la temperatura del ambiente donde viven los organismos. También se observa que la misma especie puede tener diversas tasas de mortalidad natural en diferentes áreas, dependiendo de la densidad de depredadores y competidores, cuya abundancia esta influenciada por las actividades de pesca (Sparre et al. 1994). El valor de M determinado en el presente trabajo fue superior al $\mathrm{M}=0.71$ año $^{-1}$ calculado por De Lima et al. (2004), quienes además estimaron las tasa de explotación $\left(\mathrm{E}=0.34 \mathrm{año}^{-1}\right)$, en Pernambuco, 
Brasil, demostrando que el stock de peces sufre una leve disminución debido a la pesca, alcanzando, de esta forma, una alta supervivencia de individuos con longitudes muy cercanas a la longitud máxima teórica. Billings y Munro (1974) señalan que la mortalidad natural de los Haemulidos es causada principalmente por depredación, enfermedad y vejez.

\section{AGRADECIMIENTOS}

Agradecemos a Ramona Marín†, de la Asociación de Pescadores del Sector Caracas, Boca del Río, por el apoyo brindado en la obtención de la muestra biológica. A José Luís Palazón por su ayuda en el análisis estadístico.

\section{RESUMEN}

Se determinó el crecimiento y la mortalidad natural del pez cují (Haemulon aurolineatum) del suroeste de la Isla de Margarita (julio 2005 - junio 2006), para lo cual se analizó una muestra constituida por 2541 ejemplares recolectados de la pesca artesanal de Boca del Río. La relación talla-peso entre machos y hembras no mostró diferencias significativas en las pendientes " $b$ " ( $>>0.05$; $\mathrm{ts}=-1.69)$ ni en los interceptos " $\mathrm{a}$ " ( $\mathrm{p}>0.05$; ts $=-1.01)$, por lo que se estableció una relación común para ambos sexos: $\mathrm{P}=0.038 * \mathrm{LT}^{2.87}$. A partir de los datos de distribución de frecuencia de tallas se estimó la longitud asintótica $\left(\mathrm{L}_{\infty}\right)$ aplicando la rutina de Powell-Wetherall, y el coeficiente de crecimiento (k) a través de la rutina ELEFAN I (Gayanilo et al. 1996). Posteriormente se empleó el análisis de la progresión modal, previa descomposición de la frecuencia de longitudes de acuerdo al método de Bhattacharya (1967), y se optimizaron las estimaciones de $\mathrm{L}_{\infty}$ y k según el procedimiento de Gulland y Holt (1959). Los parámetros de crecimiento estimados $\left(\mathrm{L}_{\infty}=24.2 \mathrm{~cm}\right.$ y k $=0.48$ año- $\left.{ }^{-1}\right)$ mostraron un crecimiento moderadamente rápido. Los datos de frecuencias de longitudes fueron ajustados al modelo de von Bertalanffy (1960), indicando una tendencia de tipo exponencial: crecimiento acelerado hasta los 2 años de edad, que luego se hizo lento hasta que el pez alcanzó la longitud máxima. La tasa de mortalidad natural fue alta $\left(\mathrm{M}=1.15\right.$ año $\left.^{-1}\right)$, probablemente por alta depredación.

Palabras claves: crecimiento, mortalidad, Haemulon aurolineatum, Margarita, Venezuela.

\section{REFERENCIAS}

Bertanlanffy, L. von. 1960. Principles and theory of growth, p. 137-259. In R. Musk, J. Britton \& S. Axford. 2006.
The effect of subjective fish scale ageing on growth and recruitment analyses: A case study from the UK. Acta Ichthyol. Piscat. 36: 81-84.

Bhattacharya, C. 1967. A simple method of resolution of a distribution into Gaussian components. Biometrics 23: 115-135.

Billings, C. \& J. Munro. 1974. The biology, ecology, exploitation and management of Caribbean reef fishes. Part V: The biology, ecology and bionomics of Caribbean reef fishes: Pomadasyidae (grunts). Res. Rep. 2001. Dpt. Univ. West Indies 3: 1-128

Carpenter, K. 2002. The living marine resources of the western central Atlantic. Bony fishes part 2 (Opistognathidae to Molidae), sea turtles and marine mammals. FAO Species Identification Guide for Fishery Purposes and American Society of Ichthyologists and Herpetologists Special Publication No. 5. Vols 1-3. FAO, Roma, Italia.

Cervigón, F. 1994. Los peces marinos de Venezuela. Ex Libris, Caracas, Venezuela.

Csirke, J. 1980. Introducción a la dinámica de poblaciones de peces. FAO Fish. Tech. Pap. (192.85).

Darcy, G. 1983. Synopsis of biological data on the grunts Haemulon aurolineatum and Haemulon plumieri (Pisces: Haemulidae). NOAA Tech. Rep. NMFS 448: 1-37.

De Lima, M., R. Lessa \& P. Duarte-Neto. 2004. Dinâmica de Populações e Avaliação de Estoques dos Recursos Pesqueiros da Região Nordeste. UFRPEDepartamento de Pesca. Universidade Federal Rural de Pernambuco (Coordenação da Área de Dinâmica de populações). RECIFE, Brasil.

Froese, R. \& C. Binohlan. 2000. Empirical relationships to estimate asymptotic length, length at first maturity and length at maximum yield per recruit in fishes, with a simple method to evaluate length frequency data. J. Fish Biol. 56: 758-773.

Gayanilo, F., P., Sparre \& D. Pauly. 1996. Stock assessment tools users manual. FAO, Roma, Italia.

Giménez, C., M. Ricardo \& J. Salaya. 1993. La Pesca Industrial de Arrastre. Carirubana, Caracas, Venezuela.

González, L.W. 1985. Determinación de edad y crecimiento de la sardina, Sardinella aurita Valenciennes, 1847 (Pisces: Clupeidae) de la región nororiental de Venezuela. Bol. Inst. Oceanogr. Venez. 24: 111-128. 
González, L.W. \& N. Eslava. 1999. Edad y crecimiento del pargo colorado Lutjanus purpureus Poey, 1867 (Teleostei: Lutjanidae) de la región oriental de Venezuela. Rev. Biol. Mar. Oceanogr. 34: 99-107.

Gulland, J. \& S. Holt. 1959. Estimation of growth parameters for data at unequal time intervals. J. Conseil Internat. pour L'Exploitation Mer. 25: 47-49.

Holden, M. \& D. Raitt. 1975. Manual de ciencia pesquera. Parte 2. Métodos para investigar los recursos y su aplicación. FAO Fish. Tech. Pap. (115) Rev. 1, 21 p.

Konchina, Y. 1976. Systematic and distribution of grunts (Family Pomadasyidae). Vopr. Ikhtiol. 16: 101.

Kossowski, A. 1985. Reproducción del Cují, Haemulon aurolineatum (Cuvier, 1829) (Pisces: Haemulidae) de la Isla de Margarita, Venezuela. Tesis de Grado, Licenciatura en Biología, Mención Biología Marina, Universidad de Oriente, Venezuela

Lowe-Mcconnell, R. 1999. Estudios ecológicos de comunidades de peixes tropicais. USP, São Paulo, Brasil.

Manooch, C. \& C. Barans. 1982. Distribution, abundance, and age and growth of the Tomtate, Haemulon aurolineatum, along the Southeastern United States coast. Fish. Bull. 80: 1-20.

Parra, B. 1996. Relación Longitud-peso, factor de condición e índice hepático de Haemulon aurolineatum (Cuvier: 1830) (Pisces: Haemulidae) de la Isla de Cubagua, Venezuela. Acta Cient. Venez. 47(1).

Pauly, D. 1979. Theory and management of tropical multispecies stocks: a review, with emphasis on the
Southeast Asian demersal fisheries. International Center for Living Aquatic Resources Management. Studies and review. 1: 1-35.

Pauly, D. 1983. Algunos Métodos Simples para la Evaluación de Recursos Pesqueros Tropicales. FAO Fish. Tech. Pap. (234): 49 p.

Pauly, D. \& J. Munro. 1984. Once more on the comparison of growth in fish and invertebrates. ICLARM, Fishbyte 2: 21

Powell, D. 1979. Estimation of mortality and growth parameters for the length frequency of a catch. Raport process-V Réunion Conseil International pour L'Exploitation de la Mer. 175: 167-169.

Ricker, W. 1975. Computation and interpretation of biological statistics of fish populations. Bull. Fish. Res. Board Can. 191

Sokal, R.R. \& F.J. Rohlf. 1979. Biometría. Blume, Madrid, España.

Sparre, P., E. Ursin \& S. Venema. 1997. Introduction to tropical fish stock assessment. Part 1. Manual. FAO Fish. Tech. Pap. (306.1).

Tresierra, A. \& Z. Culquichicón. 1993. Biología Pesquera. Libertad, Trujillo, Perú.

Wetherall, J. 1986. A new method for estimating growth and mortality parameters from length-frequency data. Fishbyte 4: 12-14.

Zar, J.H. 1996. Biostatistical analysis. Prentice Hall, Nueva Jersey, EEUU. 